\title{
Tangence
}

\section{Le marché aux illusions ou l'écriture du citoyen}

\section{François Dumont}

Numéro 59, janvier 1999

Écrivains d'ailleurs

URI : https://id.erudit.org/iderudit/025987ar

DOI : https://doi.org/10.7202/025987ar

Aller au sommaire du numéro

Éditeur(s)

Tangence

ISSN

0226-9554 (imprimé)

1710-0305 (numérique)

Découvrir la revue

Citer cet article

Dumont, F. (1999). Le marché aux illusions ou l'écriture du citoyen. Tangence, (59), 9-19. https://doi.org/10.7202/025987ar d'utilisation que vous pouvez consulter en ligne.

https://apropos.erudit.org/fr/usagers/politique-dutilisation/ 


\section{Le marché aux illusions ou l'écriture du citoyen}

François Dumont

Pour faire valoir l'appartenance de la prose d'idées à la littérature ou pour contester la rigidité des classements génériques, on a souvent rapproché l'essai de la forme romanesque, en soutenant qu'il peut y avoir du roman dans l'essai et de l'essai dans le roman ${ }^{1}$. De fait, l'essai peut receler une composante fictive ou narrative et le roman peut comporter une argumentation et un discours idéologique. Cela signifie-t-il que, ne faisant qu'activer à des degrés divers les mêmes virtualités, l'écriture romanesque et l'écriture essayistique soient à la limite assimilables l'une à l'autre? Un essai récent de Neil Bissoondath me paraît indiquer clairement que non; que la distinction entre fiction et intervention est parfois capitale, surtout en ce qui concerne la part de la littérature dans la définition d'une culture.

D'abord connu comme nouvelliste et romancier, Neil Bissoondath fait paraitre en 1994 un essai sur le multiculturalisme canadien, Selling Illusions, publié en français l'année suivante, dans une traduction de Jean Papineau, sous le titre Le marché aux illusions $^{2}$. On sait que la politique canadienne sur le multiculturalisme, selon les termes mêmes de la loi, "consiste à reconnaître

1 Voir par exemple André Belleau ( $Y$ a-t-il un intellectuel dans la salle?, Montréal, Primeur, 1984, p. 152-153) et Jean Marcel (Pensées, passions et proses, Montréal, l'Hexagone, 1992, p. 318-319 et 340-369). Soulignons toutefois que pour Theodor Adorno, c'est " [...] le mauvais essai [qui] raconte des personnages au lieu d'ouvrir l'accès à la chose. (Notes sur la littérature, Paris, Flammarion, 1984, p. 9).

2 Le marché aux illusions. La méprise du multiculturalisme, Montréal, Boréal et Liber, 1995. Les numéros de pages entre parenthèses renverront à cette édition. Selling Illusions. The Cult of Multiculturalism in Canada parut chez Penguin Books en 1994. Les deux romans (A Casual Brutality, 1988; The Innocence of Age, 1992) et les deux recueils de nouvelles (Digging up the Mountains, 1985; On the Eve of Uncertain Tomorrows, 1990) de Neil Bissoondath ont paru en traduction française respectivement chez Phébus (en 1992, sous les titres Retour à Casaquemada et L'innocence de l'âge) et chez Boréal (Arracber les montagnes, 1997; À l'aube de lendemains précaires, 1994). 
que le multiculturalisme reflète la diversité culturelle et raciale de la société canadienne et reconnaît la liberté de tous ses membres de maintenir, de favoriser et de partager leur patrimoine culturel, ainsi qu'à sensibiliser la population à ce fait " (cité à la page 53). Si l'orientation de cette politique paraît à première vue favoriser la diversité et la tolérance, Bissoondath insiste sur ses effets pervers: selon lui, la loi aurait plutôt tendance à accuser les différences et à favoriser la mise en place de ghettos et l'appauvrissement culturel. De sorte que le maintien artificiel des cultures d'origine, loin de contrer le racisme, le perpétuerait, étant fondé sur la même logique:

[...] reconnaittre la complexité de l'ethnicité, admettre les énormes différences qui existent à l'intérieur des groupes ethniques, aurait pour résultat de rendre l'idée et le programme du multiculturalisme absurdes. Les individus qui composent un groupe, les "ethniques" qui forment une communauté, sont souvent taillés selon plusieurs patrons très dissemblables. Des origines ethniques communes ne sont pas garantes d'unanimité de pensée. Si on ne veut pas trahir l'individu il faut s'en rapporter à une conception de l'humanité qui dépasse celle de l'ethnicité (p. 119).

En plus de cette contradiction entre l'intention déclarée d'ouverture et les conséquences effectives contraires, Bissoondath explique et illustre différents problèmes liés à la politique du multiculturalisme: celui de la double allégeance au pays d'origine et à la société d'accueil (p. 143), par exemple, ou celui de la prise en charge indue des responsabilités individuelles par l'État (p. 232), sans compter l'exposition des communautés ethniques aux manipulations politiques (p. 231).

Ces positions constituent un défi lancé autant aux discours simplistes de la rectitude politique qu'aux discours officiels subventionnés. D'un point de vue comme de l'autre, Neil Bissoondath apparaît comme une sorte de "traître (p. 18), puisque, immigrant lui-même, il semble aller à l'encontre des intérêts des immigrants: il y a dans sa position, dira-t-on, "quelque chose d'indigne. Et pourtant, souligne l'auteur, il est impossible [...] de ne pas penser à l'image du papillon épinglé par des mains prudentes et aimantes convaincues de servir la gloire des... papillons" (p. 56). Refusant de se considérer comme le représentant d'une minorité ou de quelque groupe que ce soit, Bissoondath estime que "la lutte aux stéréotypes, qui sont à l'origine de toutes 
les formes de racisme, [est] une affaire très personnelle. [...] aucun appareil bureaucratique ne pourra jamais mettre fin aussi efficacement à l'usage de stéréotypes et à l'enfermement dans les rôles sociaux qu'une récusation immédiate de la part de ceux qui s'y opposent" (p. 222). Et la meilleure façon, pour l'individu, de faire valoir son autonomie serait de sortir des problématiques binaires préfabriquées. L'alternative que Bissoondath récuse avec le plus d'insistance est l'opposition entre multiculturalisme et racisme. Tout en soutenant que le multiculturalisme accentue les frontières ethniques, il plaide pour la diversité, mais une diversité fondée sur la singularité des voix: "Une société libre repose sur la diversité des voix et des conceptions de ceux qui s'y font entendre autant que sur les débats d'opinion. On perdrait au change à vouloir réduire cette diversité" (p. 187). En critiquant le multiculturalisme, l'auteur ne devient donc pas pour autant un apôtre de l'homogénéisation de la culture, bien au contraire.

Du point de vue des rapports entre le multiculturalisme et la "question constitutionnelle", la distance entre les positions de Bissoondath et les perspectives prévisibles est accentuée par le passage de la langue anglaise à la langue française. À cet égard, les effets de traduction sont nombreux. Si, par exemple, pour un lecteur du Canada anglais, il y avait quelque originalité à se voir présenter à plusieurs reprises le Québec comme un modèle pour le Canada (p. 207, p. 219, p. 229...), pour un lecteur francophone du Québec, il est non moins curieux de se faire initier au Québec par des déclarations traduites du Globe and Mail (p. 203, p. 210, p. $214 \ldots)^{3}$. Ce curieux déphasage est l'occasion d'un recul qui fait clairement apercevoir la divergence des interprétations du multiculturalisme, lu tantôt comme "l'instrument grâce auquel on voudrait gommer l'arrogance historique du Canada anglais à l'égard du Québec" (p. 75), tantôt exactement en sens inverse (p. 209). Encore une fois, Bissoondath se tient à distance des

3 Par ailleurs, la candeur de l'auteur est sans doute aussi déconcertante pour le lecteur canadien-anglais que pour le lecteur québécois francophone, lorsque l'essayiste propose "le renforcement des conquis parallèlement à l'affaiblissement des conquérants jusqu'à ce qu'on atteigne un certain équilibre. (p. 74)... Enfin, si la critique du multiculturalisme trouve un écho sympathique chez plusieurs Québécois qui se méfient de l'impact de cette politique sur le statut du Québec, la mise à l'écart de l'insulte et du mépris n'est sans doute pas étrangère non plus au relais assumé par le traducteur, les éditeurs et la préfacière. 
12

alternatives: sa position n'est assimilable ni au point de vue fédéraliste dominant ni, pour un lecteur québécois, au point de vue indépendantiste habituel. Mais, plus encore peut-être que cette distance par rapport aux schémas binaires, c'est l'aménagement d'un espace distinct pour la littérature qui constituerait le véritable fondement du livre, ou du moins, comme le souligne la préface de Lise Bissonnette, "son origine" (p. 11).

En effet, avant la publication de son essai, on a reproché à Neil Bissoondath de ternir l'image des minorités dans ses nouvelles et ses romans, par des descriptions trop dures à leur égard et surtout par son refus implicite de se faire par la fiction leur apologiste ou leur porte-parole. C'est le cas de Dionne Brand: "En se servant d'un Neil Bissoondath pour dénoncer la critique de l'appropriation culturelle, écrivit-elle, l'establishment culturel blanc se sert d'un écrivain issu des minoritês visibles pour faire taire et discréditer tous les autres membres de ces communautés et, par la même occasion, pour confirmer la représentation coloniale essentielle à la domination raciale et lui rendre une certaine crédibilité" (cité p. 179). Ce raisonnement ne fait pas que reproduire la logique de l'alternative entre multiculturalisme et racisme. Il présuppose de surcroît que l'œuvre littéraire est une prise de position idéologique au même titre que la déclaration politique. D'où la double dimension du Marché aux illusions: remise en question du multiculturalisme et défense de l'autonomie de la littérature.

Dès les premières pages et jusqu'à l'avant-dernier chapitre, la quasi-absence de la littérature est frappante. Cela ne manque pas d'étonner, puisque l'œuvre de l'auteur ne compte, au moment de la parution de l'essai, que des recueils de nouvelles et des romans. Plutôt que de se présenter d'emblée comme un écrivain, il commence par définir sa parole d'essayiste par la négative: "Je ne suis ni docteur en littérature, ni sociologue, ni politicien. Je ne prétends pas détenir toutes les réponses, ni même une seule [...] Il s'agit pour moi de contribuer à une discussion nécessaire [...]” (p. 21). C'est donc en tant que citoyen, abstraction faite jusqu'au chapitre cinq de son expérience d'écrivain, que l'auteur expose ses idées. Non sans livrer par la même occasion son point de vue sur la variété des discours qui orientent la réflexion et l'action du citoyen. Quatre types de discours sont mis en scène avant que la question de la spécificité de la littérature ne soit abordée: le témoignage, l'actualité, le discours des experts et le discours politique. 
L'auteur évoque d'abord son enfance à Trinidad, surtout marquée par l'apprentissage du "sens de l'ironie" (p. 26), prise de distance par rapport au milieu, aux idéologies et au vécu luimême. C'est par l'ironie ${ }^{4}$ que l'enfant découvre notamment l'écart entre "le mensonge soigneusement entretenu d'une vie harmonieuse" (p. 29) et les divisions ethniques. À son propre témoignage, l'auteur ajoute celui de membres de sa famille, d'amis et de connaissances. Mais c'est le journal qui figure de loin comme la source principale. Aux témoignages s'ajoutent un très grand nombre de faits divers (souvent résumés en enfilade, ainsi qu'ils se présentent dans le journal et dans les bulletins de nouvelles). Ces anecdotes servent à illustrer des enjeux politiques et à proposer certaines nuances à partir de contre-exemples, alors que c'est le recours au raisonnement qui apparaît toujours comme l'élément déterminant. Car les limites du monde des faits divers et de la parole du journaliste sont souvent soulignées: distorsion des faits (p. 60), amplification de phénomènes marginaux (p. 62), promotion facile d'idées de droite (p. 80), notamment. Mais les critiques de l'essayiste n'épargnent pas pour autant le discours apparemment plus solide de l'expert.

L'évocation de disciplines est en effet toujours assortie de remarques critiques. Ainsi le "jargon" du droit est associé aux "incantations dans les sectes religieuses" (p. 56); les abstractions de la philosophie devraient être "tempérées par l'expérience" (p. 76); les statistiques «s'adaptent facilement à n'importe quelle besogne" (p. 114); quant aux "versions officielles de l'histoire ", il faut les tenir pour "suspectes" (p. 213). Mais le reproche que Bissoondath adresse le plus souvent aux experts est leur démission devant les problèmes de l'existence commune. L'historien Michael Ignatieff, sur lequel Bissoondath s'appuie à plusieurs reprises, est présenté comme une sorte de modèle a contrario d'alliance entre connaissance et humanité; comme un

historien que sa formation universitaire n'a pas privé du sens des événements, du sens d'une histoire où les hommes et les femmes occupent une place centrale et façonnent les événements autant qu'ils sont façonnés par eux. Si le journalisme est la chronique brute des "faits", et si l'histoire est la mise en ordre

4 Plus loin (p. 121), l'ironie est explicitement liée à la littérature, ce qui permet de considérer à rebours cet apprentissage comme celui de la littérature ellemême. 
14

réfléchie de notre compréhension de ces mêmes "faits", alors l'histoire telle que la pratique Michael Ignatieff se situe quelque part après le moment où les passions se sont refroidies mais avant celui où la poussière retombe (p. 46).

Encore une fois, l'auteur plaide pour l'irréductibilité de l'individu (auquel l'historiographie doit faire une place, qu'il s'agisse de la liberté des acteurs ou de la subjectivité de l'historien). Ailleurs, il insiste aussi sur la fonction du scientifique qui est de remettre en question les préjugés des individus et des groupes, ce qui "doit comporter le droit de déranger et même celui d'offenser" (p. 183). En l'occurrence, il s'agit du devoir de résister aux censures de toutes sortes, qu'elles soient de droite ou de gauche.

Pour Bissoondath, le discours politique est miné des deux côtés. Le discours de droite est rapidement écarté, à titre de "litanie de peurs rationalisées" (p. 80). Les positions radicales de la gauche sont plus longuement commentées. Bissoondath fustige d'abord la gauche qui "n'encourage pas l'exercice de l'intelligence, mais manifeste son abdication, [qui] n'incite pas à l'invention d'idées nouvelles, mais cherche les aveux de confessionnal" (p. 170). Il s'agit donc d'être nuancé, inventif, libre par rapport aux réponses préfabriquées des discours politiques dominants, qu'ils appartiennent aux extrêmes figés de la droite et de la gauche ou au radicalisme parfois tout aussi désespérant de la bureaucratie (p. 69). La nécessité des nuances, de l'invention et de l'exercice de la liberté commanderait de tenir l'idée de révolution à distance:

Les déséquilibres économiques et sociaux ne se corrigent pas du jour au lendemain. Seule une révolution peut entraîner un changement aussi radical, et si l'histoire du $\mathrm{xx}^{e}$ siècle nous a appris une chose, c'est que le changement révolutionnaire est illusoire: c'est seulement les oppresseurs et la nature de l'oppression qui se transforment. Un véritable changement ne peut donc être imposé; il doit se produire lentement, avec le temps et l'expérience, de l'intérieur (p. 198).

L'«intérieur" en question renvoie à l'intériorité de l'individu, ou plutôt d'un sujet en relation égalitaire avec d'autres sujets: le citoyen tel que l'incarne Bissoondath dans son essai. Mais en quoi l'"expérience" et l'uintérieur" auxquels il en appelle sont-ils liés à la littérature?

Il faut d'abord souligner que le regard critique porté sur les discours du témoignage, de l'actualité, de la science et de la poli- 
tique n'épargne pas la littérature elle-même. Si le "romanesque" est parfois présenté comme une sorte d'antidote à la manie scientifique des "types" (p. 44), la littérature constitue aussi une menace, notamment sous la forme d'un "romantisme" trompeur. À ce sujet, l'auteur cite Nino Ricci qui observe que plusieurs immigrants

restent attachés à une vision romantique de leur pays qui a pourtant radicalement changé depuis leur départ, et cela contribue à une distorsion de leur identité. S'ils y avaient vécu, ils auraient suivi l'évolution de leur pays. Ici, ils s'accrochent à une vision idéalisée de leur culture traditionnelle (cité à la p. 141).

Reprenant plus loin cette idée, Bissoondath insiste sur le fait que le monde de la littérature, à titre de représentation "thêatrale", illusoire, peut s'avérer nuisible, non seulement pour l'immigrant en particulier, mais encore pour le citoyen en général:

Il est facile, grâce à la mémoire sélective, d'oublier que tout a changé; il est également facile de commettre l'erreur de s'abandonner à la nostalgie aiguë d'une certaine perfection qui vit dans notre souvenir. En mettant l'accent sur le théâtral, le multiculturalisme contribue à accréditer de telles illusions et, une fois de plus, ce sont l'individu et l'État qui y perdent - l'un en s'accrochant à ses illusions et en se laissant parfois guider par elles, l'autre en perdant tout son attrait à côté des illusions qu'on dit être la réalité (p. 231).

Le monde de la fiction - d'une certaine fiction, à tout le moins - est ici l'adversaire par rapport auquel le citoyen prend ses distances. Mais on lit aussi, à l'inverse, de multiples éloges de la fiction: le romancier plaidera souvent pour la liberté d'écriture dans le registre qui est le sien, en recourant par exemple à cette maxime de Salman Rushdie: "Plus l'imagination est libre, mieux elle fonctionne" (cité p. 157), et surtout en insistant pour libérer l'écrivain des rôles "paralittéraires" qui sont non seulement étrangers mais contraires à la spécificité de sa parole: "Propagandiste, publicitaire, porte-parolen (p. 165). Ce qui n'empêche pas que la littérature tienne le rôle d'un guide pour l'action. C'est notamment le cas des pièces de Shakespeare, considérées par l'essayiste comme des fables, par exemple sur l'autonomie de l'individu par rapport à ses origines ("Roméo et Juliette sont morts parce que leur foi et leur imagination ont été trahies par les passions de leurs parents", p. 136) ou sur la fonction de la littérature (lecture du Marchand de Venise: "Nous ne sommes pas obligés d'aimer les brutes qui nous entourent, ni même de leur accorder le 
16

pardon, mais c'est à nos risques que nous choisissons de ne pas voir leur humanité", p. 161).

D'un certain côté il y a donc, dans Le marché aux illusions, une "division du travail" assez nette qui ordonne en complémentarité la responsabilité du citoyen et la liberté de l'écrivain. Cependant, la correspondance entre l'attitude du premier et la manière du second est à certains moments frappante. Par exemple, l'empathie de l'essayiste pour les individus s'avère conforme à l'empathie du romancier pour ses personnages, lorsque l'essayiste cherche patiemment les fondements existentiels de positions idéologiques indéfendables (p. 81, p. 116, p. 161...). L'essayiste met alors en pratique cette "suspension du jugement moral "qui caractérise selon Milan Kundera l'art du romancier ${ }^{5}$, et en même temps ce conseil que jeune homme il reçut de son oncle V. S. Naipaul, et qui s'applique autant à l'écriture qu'à l'existence: d'abord "essayer de comprendre" les individus (p. 220). Il s'éloigne de la sorte du registre de l'insulte qui à son avis empoisonne bien des débats contemporains : avec la "simplicité injurieuse", résume-t-il, on ne peut qu'"obscurci[t] la vraie complexité de la question" (p. 89). L'insulte serait le contraire même de l'"offense" salutaire de la pensée et de la littérature. S'il y a une leçon à tirer de l'expérience de l'écrivain pour le citoyen, ce serait avant tout de savoir résister aux simplifications - par l'interrogation empathique, mais aussi par l'ironie (p. 116), lesquelles convergent vers la "démystification de l'Autre" (p. 193).

Avec l'empathie et l'ironie, l'attention aux faits concrets se déplace aussi du roman à l'essai. Bissoondath fait indéniablement partie de ce type de romanciers dont parle Philip Roth, pour qui " tout événement de pure imagination trouve son origine là, dans les faits, dans le particulier et non dans le philosophique, l'idéologique ou l'abstrait. "6. Le fondement même du Marché aux illusions est d'opposer la réalité concrète au philosophique, à l'idéologique et à l'abstrait - d'où le recours si fréquent aux faits divers. Mais dans l'essai, le caractère représentatif des faits est discuté, l'idéologie à combattre ou à nuancer et la position du citoyen sont explicitées; alors que la fiction narrative reste au seuil

5 Voir notamment "Le jour où Panurge ne fera plus rire", dans Les testaments trabis, Paris, Gallimard, 1993.

6 Philip Roth, Les faits. Autobiograpbie d'un romancier, Paris, Gallimard, 1990, p. 13 . 
de la discussion et de la prise de position, tout en mettant en lumière le caractère irréductible de l'expérience. Cette différence apparaît nettement si on compare par exemple l'utilisation que font l'essayiste et le nouvelliste du cas de l'immigrant d'origine antillaise qui réclame le droit de fêter dans le bruit au nom du caractère sacré de sa culture. Dans l'essai, ce cas permet de poser la question des "limites de la diversité" (p. 147-148), alors que la nouvelle ${ }^{7}$ est axée sur un désarroi qui est décrit sans être expliqué ou commenté, sinon du point de vue des personnages euxmêmes. Neil Bissoondath insiste du reste à plusieurs reprises, dans son essai, sur l'autonomie des personnages romanesques, autant par rapport à l'écrivain (ils "ne sont pas les jouets des auteurs", p. 191) que par rapport à une éventuelle représentativité ("un personnage ne représente que lui-même", p. 192). Ainsi, le romancier, devenant essayiste, s'il peut adapter certains aspects de son écriture, doit surtout préserver la spécificité des deux genres.

On a beaucoup mis l'accent, depuis quelques années, sur la nécessaire distinction entre le sujet empirique et le sujet de l'écriture. Inversement, on a voulu rapprocher les genres littéraires, au point de les considérer comme des conventions purement arbitraires. L'exemple de l'œuvre de Bissoondath montre que les deux points de vue peuvent être liés, et qu'ils doivent tous les deux être nuancés. La question centrale me paraît être ici celle de la nature de la fiction, autant en ce qui concerne le sujet que le genre. La différence fondamentale entre la parole du citoyen et celle du romancier comme entre l'essai et le roman (du moins dans l'économie de l'œuvre de Bissoondath) s'éclaire à partir du point de vue théorique adopté par Karlheinz Stierle: "Indépendamment de tous les rapports singuliers qui s'y marquent à la réalité, observe-t-il, la caractéristique essentielle d'un texte de fiction est d'être une assertion non vérifiable. " ${ }^{8}$ À partir de ce constat, le théoricien rejoint par la logique les positions de Bissoondath sur l'autonomie de l'écriture romanesque:

Par principe, la fiction ne se laisse pas corriger par une connaissance plus exacte des faits auxquels elle se rapporte. Alors que tout texte référentiel se laisse corriger par la réalité, le texte de

7 Il s'agit de la nouvelle intitulée "Danser", dans Arracber les montagnes, op. cit., p. 231-256.

8 Karlheinz Stierle, "Réception et fiction", Poétique, n 39, septembre 1979, p. 299. 
fiction n'est tel que s'il met en jeu un écart (qui n'est pas à corriger mais seulement à interpréter ou à critiquer) par rapport au donné $[\ldots]$ ?

Voilà un aspect qu'à mon avis la théorie de l'essai n'a pas encore suffisamment considéré ${ }^{10}$. S'il est vrai que la narrativité peut marquer autant la prose d'idées que la fiction romanesque ${ }^{11}$, il s'avère par ailleurs abusif, en ce qui concerne l'œuvre de Bissoondath, de considérer le "je" de l'essayiste comme un pur équivalent du narrateur romanesque. En fait, ici, précisément, la distinction entre le monde de la fiction, qui ne peut pas être corrigé, et le monde réel, qui doit l'être, détermine les rôles respectifs du romancier et du citoyen. Il ne s'agit pas de paroles forcément étrangères en tous points; mais sur le plan de la fiction, elles diffèrent radicalement, puisque l'une est de l'ordre de l'autonomie et de l'exploration, l'autre de l'intervention et de l'élucidation ${ }^{12}$.

9 Loc. cit. Voir aussi Logique des genres littéraires, de Käte Hamburger (Paris, Seuil, 1986 [1957]), où la théoricienne, à partir de la notion de fiction, exclut carrément l'essai du système de la littérature.

10 Lorsque la question de la dimension fictive de l'essai est abordée, plusieurs théoriciens ou praticiens du genre parlent de son caractère fictionnel comme d'une indiscutable évidence. André Belleau écrit par exemple: "Il semble y avoir accord de principe sur le fait que l'essai procède du fictionnel à l'instar de tout discours littéraire "(op. cit., p. 150), tandis que Jean Larose peut écrire dans l'un de ses essais: "Il faut aussi comprendre l'idée de fiction, je dirais disposer d'assez de jeu souverain pour sentir la fiction, savoir suspendre la décision du sens le temps d'un jeu, ou d'un essai littéraire... (La souveraineté rampante, Montréal, Boréal, 1994, p. 56-57). De son côté, Jean Marcel, après avoir parlé de "JE non métaphorique" (op. cit., p. 318), s'empresse de se corriger, en soutenant que "le JE fondateur du discours essayistique n'est pas moins "construit" et "fictionnel" que le JE générateur du récit romanesque seulement, ajoute-t-il, il l'est autrement" (ibid., p. 342). Cette position nuancée me paraît plus fertile que la position plus tranchée de Belleau et Larose, reprise en ces termes par Robert Vigneault: "[la] mise en texte, qui caractérise le discours de l'essai, procède, comme tout discours littéraire, de l'activité fictionnelle de l'imagination. (L'écriture de l'essai, Montréal, l'Hexagone, 1994, p. 35). Rappelons enfin que selon les vues du jeune Lukács dans L'âme et les formes (Paris, Gallimard, 1974), le "je " de l'essayiste transcende la vie empirique sans être pour autant assimilé au "je" romanesque.

11 Ou tout autre genre (du discours ou de la littérature), comme le montre par exemple Micheline Cambron dans Une société, un récit, Montréal, l'Hexagone, 1989.

12 Évidemment, d'autres poétiques sont concevables, y compris la juxtaposition de ces fonctions. Dans certains cas (chez Jean Larose, par exemple), la frontière entre la position idéologique de l'intellectuel et l'exploration de l'écrivain est toutefois très ambiguë. 
Cette distinction est explicite dans Le marcbé aux illusions, sans doute parce que le romancier a souvent été pris à partie par des critiques qui voyaient dans la fiction un monde malléable et corvéable. L'essai de Bissoondath, par son propos et par son exemple, montre que si l'écrivain doit s'engager, c'est au même titre et sur le même terrain que le citoyen; à ce moment il reste un écrivain, mais il sort de la fiction. De ce point de vue, on pourrait d'ailleurs avancer que l'erreur théorique fondamentale du multiculturalisme serait de croire que l'art en particulier (mais aussi la culture en général) peut être programmé. Or, dans cette logique, quel est le rôle du citoyen en ce qui a trait à la définition de sa culture? Neil Bissoondath affirme à plusieurs reprises qu'il faut remplacer la politique multiculturaliste par "une nouvelle représentation de nous-mêmes" (p. 86); qu'il s'agit "d'élaborer une nouvelle conception de nous-mêmes" (p. 234). Mais il ne fait pas de proposition. L'art pourrait-il constituer à cet égard un point de départ ${ }^{13}$ ? Si oui, dans la perspective du Marché aux illusions, ce ne serait que par l'interprétation et la critique de ce que soumet librement l'art; c'est-à-dire par l'essai.

13 On peut penser ici à la notion de littérature nationale. En effet, lorsque Bissoondath découvre le Québec, c'est d'abord à travers sa littérature (p. 204). Toutefois, dans un entretien récent, lorsque Marcos Ancelovici et Francis Dupuis-Déri lui demandent s'il croit à "l'existence d'une littérature nationale", il répond par la négative, avant de concéder que "quelques références sont nécessaires", même si "elles ne renvoient pas au coulu des choses". Il semble que pour lui le danger "de croire à ses propres stéréotypes "soit beaucoup plus important que la nécessité de circonscrire une culture commune (voir Marcos Ancelovici et Francis Dupuis-Déri, L'arcbipel identitaire, Montréal, Boréal, 1997, p. 151-167). Ajoutons que la notion de "littérature migrante", de plus en plus souvent présentée comme une catégorie de la littérature canadienne ou québécoise, apparaît, dans la perspective de Neil Bissoondath, au mieux comme une tautologie, au pire comme une manifestation de l'efficacité de la politique du multiculturalisme. 